\title{
Clinical aspects of short-chain acyl-CoA dehydrogenase deficiency
}

\author{
Bianca T. van Maldegem • Ronald J. A. Wanders • \\ Frits A. Wijburg
}

Received: 21 October 2009 /Revised: 8 March 2010 / Accepted: 12 March 2010 /Published online: 29 April 2010

(C) The Author(s) 2010. This article is published with open access at Springerlink.com

\begin{abstract}
Short-chain acyl-CoA dehydrogenase deficiency (SCADD) is an autosomal recessive inborn error of mitochondrial fatty acid oxidation. SCADD is biochemically characterized by increased C4-carnitine in plasma and ethylmalonic acid in urine. The diagnosis of SCADD is confirmed by DNA analysis showing SCAD gene mutations and/or variants. SCAD gene variants are present in homozygous form in approximately $6 \%$ of the general population and considered to confer susceptibility to development of clinical disease. Clinically, SCADD generally appears to present early in life and to be most frequently associated with developmental delay, hypotonia, epilepsy, behavioral disorders, and hypoglycemia. However, these symptoms often ameliorate and even disappear spontaneously during follow-up and were found to be unrelated to the SCAD genotype. In addition, in some cases, symptoms initially attributed to SCADD could later be
\end{abstract}

Communicated by: Verena Peters

References to electronic databases: Online Mendelian Inheritance in Man: http://www.ncbi.nlm.nih.gov/sites/entrez.

Enzyme commission: www.chem.qmul.ac.uk/iubmb/enzyme/.

Competing interests: None declared.

Presented at the Fulda-Symposium Fatty Acid

Oxidation: Clinical, Biochemical and Molecular Aspects, 12-14

November 2008

B. T. van Maldegem $(\varangle) \cdot$ F. A. Wijburg

Department of Pediatrics, Academic Medical Center,

University of Amsterdam,

Meibergdreef 9,

1105 AZ Amsterdam, The Netherlands

e-mail: b.van.maldegem@gelre.nl

\section{R. J. A. Wanders}

Laboratory Genetic Metabolic Diseases,

Academic Medical Center, University of Amsterdam,

Amsterdam, The Netherlands explained by other causes. Finally, SCADD relatives of SCADD patients as well as almost all SCADD individuals diagnosed by neonatal screening remained asymptomatic during follow-up. This potential lack of clinical consequences of SCADD has several implications. First, the diagnosis of SCADD should never preclude extension of the diagnostic workup for other potential causes of the observed symptoms. Second, patients and parents should be clearly informed about the potential lack of relevance of the disorder to avoid unfounded anxiety. Furthermore, to date, SCADD is not an optimal candidate for inclusion in newborn screening programs. More studies are needed to fully establish the relevance of SCADD and solve the question as to whether SCADD is involved in a multifactorial disease or represents a nondisease.

\author{
Abbreviations \\ SCAD Short-chain acyl-CoA dehydrogenase \\ SCADD Short-chain acyl-CoA dehydrogenase deficiency \\ FAO Fatty acid oxidation \\ C4-C Butyryl carnitine \\ C4-CoA Butyryl-CoA \\ EMA Ethylmalonic acid \\ $A C A D S$ SCAD encoding gene
}

\section{Introduction}

Short-chain acyl-CoA dehydrogenase (SCAD, EC 1.3.99.2) deficiency (SCADD, OMIM 201470) is an autosomal recessive inborn error of mitochondrial fatty acid oxidation (FAO). SCAD catalyzes the dehydrogenation of butyryl$\mathrm{CoA}(\mathrm{C} 4-\mathrm{CoA})$ during the first step of the short-chain fatty acid $\beta$-oxidation spiral. Impaired SCAD activity results in accumulation of its substrate $(\mathrm{C} 4-\mathrm{CoA})$ and the subsequent production of alternative metabolites, including the following: 
(1) the corresponding carnitine-ester, i.e., butyryl carnitine (C4-C), (2) the corresponding glycine-ester (butyryl glycine), (3) butyrate, and (4) ethylmalonic acid (EMA). C4-C, measured in blood, and EMA, measured in urine, are generally used as biochemical markers for SCADD. The diagnosis of SCADD is usually confirmed by DNA analysis. The majority of SCADD patients are homozygous or compound heterozygous for two common variants of the SCAD encoding gene (ACADS), or for ACADS variants in combination with an inactivating mutation (Waisbren et al. 2008; Pedersen et al. 2008; van Maldegem et al. 2006; Tein et al. 2008). Up to 70 different inactivating $A C A D S$ mutations have been reported so far (Naito et al. 1990; Gregersen et al. 2008; van Maldegem et al. 2006; Corydon et al. 2001; Gregersen et al. 1998; Koeberl et al. 2003; Seidel et al. 2003; Kristensen et al. 1994; Pedersen et al. 2008; Jethva et al. 2008; Waisbren et al. 2008). There is a remarkably high prevalence of homozygosity for $A C A D S$ variants in the general population, with frequencies of approximately $0.3 \%$ for the p.R171W (c.511C > T) and 5.5\% for the p.G209S (c.625G > A) variant (Maldegem et al. 2005; Nagan et al. 2003). Homozygosity for these variants is considered to confer susceptibility to clinical disease (Corydon et al. 2001; Gregersen et al. 1998; Gregersen et al. 2000). Most SCADD patients have been diagnosed as a result of investigations for neurological symptoms and/or hypoglycemia (Waisbren et al. 2008; van Maldegem et al. 2006; Tein et al. 2008; Pedersen et al. 2008). There is, however, debate on the clinical relevance of SCADD (van Maldegem et al. 2006; Dietzen et al. 2009). Nevertheless, newborns are screened for SCADD in the USA [National Newborn Screening Status Report (NNSGRC) (2008) Updated 07/07/09].

In this manuscript, we summarize the clinical aspects of SCADD and discuss the clinical relevance of this inborn error of metabolism.

\section{Clinical symptoms in SCADD}

Symptoms in clinically identified patients

The first SCADD patient was originally reported by Amendt and coworkers (Amendt et al. 1987) and subsequently genetically confirmed by Naito in 1990 (Naito et al. 1990). The patient was reported to suffer from lethargy, hypertonia, and circulatory problems with metabolic acidosis during her first week of life. Although she was reported to show normal growth and development without recurrence of metabolic acidosis up to the age of 2 years, Bhala et al. later reported that this patient had died, without reporting clinical details (Bhala et al. 1995). As this publication included another SCADD patient who died after initial presentation with severe skeletal muscle hypotonia, a devastating clinical course of SCADD was suggested. However, the same publication included another two patients who initially presented with "possible hyperactivity" and "probable seizure activity" but with a normal follow-up. Initially, a few other patients with SCADD had been reported (Bhala et al. 1995; Amendt et al. 1987; Coates et al. 1988), however in these patients, the diagnosis was, to our knowledge, not genetically confirmed. As the definitive diagnosis of SCADD requires molecular testing (van Maldegem et al. 2006), these patients are excluded from this review.

Subsequently, a large cohort of SCADD patients was presented by Corydon et al., and several case reports were published (Corydon et al. 2001; Baerlocher et al. 1997; Bok et al. 2003; Birkebaek et al. 2002; Kmoch et al. 1995; Matern et al. 2001; Kurian et al. 2004). Based on these publications, SCADD appeared to be associated with a wide spectrum of clinical signs and symptoms, including developmental delay, hypotonia, epilepsy, and hypoglycemia, and in solitary cases dysmorphic features, vomiting, failure to thrive, hepatic dysfunction after premature delivery, and bilateral optic atrophy. One case report suggested the association between SCADD and acute fatty liver of pregnancy in the mother. Again, a striking spectrum was observed in patient outcome, which was reported for seven patients of whom five fully recovered, one slowly progressed, and one died (Kurian et al. 2004; Birkebaek et al. 2002; Bok et al. 2003; Matern et al. 2001; Seidel et al. 2003; Kmoch et al. 1995).

In 2006, we reported data on 31 Dutch SCADD patients (van Maldegem et al. 2006). The most frequently reported symptom in this cohort was developmental delay, followed by epilepsy, behavioral disorders, and hypoglycemia. Behavioral disorders, observed in eight out of the 31 patients, had not been previously reported in SCADD patients. Remarkably, most of the clinically severely affected patients belonged to the group of patients homozygous for the c. $625 \mathrm{G}>\mathrm{A}$ variant. In four patients, additional diagnoses that were highly likely to be causing the clinical symptoms were made after the initial diagnosis of SCADD.

In 2008, Tein and coworkers published a study on ten SCADD patients (Tein et al. 2008) in whom developmental delay was again the most common symptom, but this time, hypotonia was also as frequent. In addition, this study reported for the first time a relatively high prevalence of lethargy (five patients), myopathy (four patients), and facial weakness (three patients). All patients in this study were of Ashkenazi Jewish descent, carried the $c .319 \mathrm{C}>\mathrm{T}$ mutation, and were either homozygous for this mutation or had the c. $625 \mathrm{G}>\mathrm{A}$ variant on the other allele. In two patients with myopathy, a muscle biopsy revealed multiminicore disease, a rare congenital myopathic disorder. However, other genetic causes for multiminicore disease, in particular, mutations in SEPN1 and RYR1, which are present in about 
$50 \%$ of cases (Jungbluth 2007), had not been excluded. In the same year, Pedersen and coworkers published a study on a very large cohort of 114 SCADD patients from Europe, New Zealand, and Canada. Again developmental delay was the most frequently reported clinical sign, in combination with hypotonia, seizures, and failure to thrive (Pedersen et al. 2008). In addition, several patients were reported to have failure to thrive and hypotonia without developmental delay, and a smaller group had dysmorphic features. A third study, also published this same year, by Waisbren and coworkers, reported on another six clinically identified patients (Waisbren et al. 2008). In three of them, newborn screening failed to detect SCADD, and in the other three, no screening for SCADD was performed. The most significant symptoms in these six patients were developmental delay, hypotonia, feeding problems, failure to thrive, and epilepsy.

Age of presentation, symptom transience, and genotype-phenotype relation in clinically identified patients

It appears that clinical signs and symptoms in SCADD patients generally present early in life, with almost all patients presenting under the age of 5 years (Pedersen et al. 2008; Tein et al. 2008; van Maldegem et al. 2006; Waisbren et al. 2008). Symptoms were transient in nine of the 31 patients in the van Maldegem study and two of the six patients in the Waisbren study. Furthermore, no association could be made between genotype and clinical phenotype in the patients from the Pedersen and the van Maldegem study.

\section{Clinical symptoms in SCADD individuals identified} by newborn screening

With the implementation of newborn screening for SCADD in the USA and Australia, the clinical spectrum of SCADD has expanded. Several follow-up studies of SCADD newborns diagnosed through newborn screening were published within the last few years. The first one reported 17 SCADD newborns who all remained symptom free at follow-up during their first 2 years of life (Rhead et al. 2002). Of the three patients reported by Koeberl et al. (2003), one developed seizures and a cerebral infarction at the age of 10 weeks, and the other two remained symptom free during their first 3 years of life. One of the eight children reported by Waisbren and co-workers (2008) showed developmental delay consisting of a language delay at the age of 2 years. Jethva and Ficicioglu reported a group of 14 children with SCADD, of whom 11 were identified by newborn screening and three diagnosed by screening of sibs diagnosed through newborn screening. During a follow-up of 1-7 years, in two of them (siblings), speech delay was diagnosed. A causative relationship between this speech delay and SCADD was considered unlikely, as both parents had learning disabilities, suggesting other causes (Jethva and Ficicioglu 2008). All four Australian SCADD children diagnosed by newborn screening and studied during 6 years remained symptom free. SCADD has meanwhile been excluded from the Australian screening panel because of supposed lack of clinical significance (Wilcken 2008).

An interesting finding by Waisbren and coworkers is a relatively high prevalence of pregnancy complications in mothers of children diagnosed with SCADD by newborn screening as well as in mothers of the group of SCADD patients diagnosed because of clinical symptoms (Waisbren et al. 2008). In five out of 14 patients, hypertension, maternal bradycardia, pre-eclampsia, and mild hemolysis, elevated liver enzyme levels, and a low platelet count (HELLP syndrome) were reported. This suggests that fetal SCADD, like other FAO disorders such as long-chain 3hydroxyacyl-coenzyme A dehydrogenase (LCHAD) deficiency, might be associated with pregnancy complications, as previously suggested (Matern et al. 2001). However, in almost $30 \%$ of all pregnancies in the Dutch population, mild to moderate pregnancy complications were reported, including hypertensive disorders and pre-eclampsia (Dutch Hospital Database 2009). Furthermore, the association between
Fig. 1 Comparison of the number of symptoms in metabolically screened patients to the number of symptoms at presentation in the Dutch shortchain acyl-CoA dehydrogenase deficiency (SCADD) patients group
Metabolically screened patients

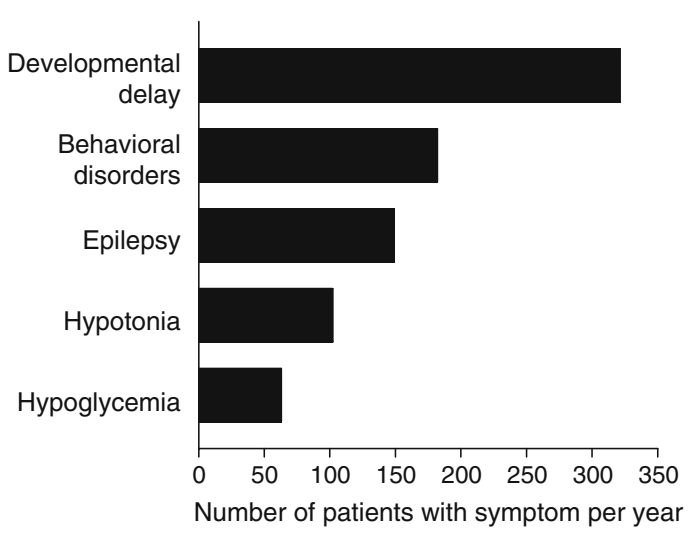

Dutch SCADD patients

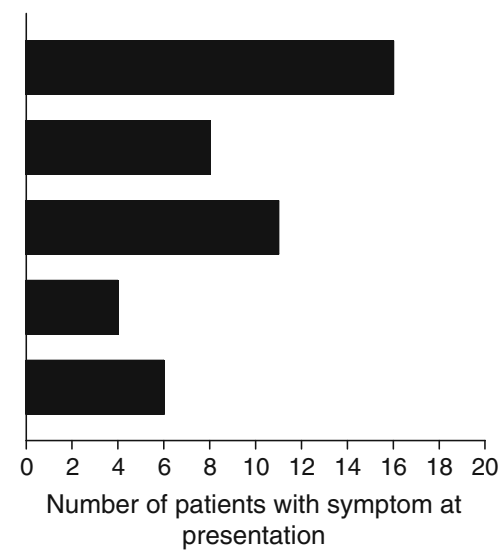


SCADD and maternal pregnancy complications was not detected in the Dutch cohort of SCADD patients (van Maldegem et al. 2006), as maternal disease during pregnancy was only reported in four out of 24 patients (17\%) for whom pregnancy details were known (unpublished data).

Family studies

Thirty-seven relatives (20 parents and 17 sibs) of the SCADD patients from the Dutch cohort were investigated for their $A C A D S$ genotype, and nine of them were found to have the same $A C A D S$ genotype as the proband (van Maldegem et al. 2006). Except for one father with an $A C A D S$ genotype homozygous for the c. $625 \mathrm{G}>\mathrm{A}$ variant, all relatives with an $A C A D S$ genotype identical to the proband were found to have increased C4-C and/or EMA. Eight relatives had always been healthy, whereas one had a history of transient food refusal during her first year of life.

\section{Clinical significance}

The clinical studies discussed above show that symptoms in SCADD generally present early in life and most frequently concern developmental delay, hypotonia, epilepsy, behavioral disorders, and hypoglycemia. Signs and symptoms often ameliorate and disappear completely during follow-up. In addition, they can sometimes be explained by other causes and are not related to the ACADS genotype. Finally, almost all relatives diagnosed with SCADD, as well as almost all individuals detected by newborn screening, remain fully asymptomatic. Based on these observations, one may question the clinical relevance of SCADD. Indeed, we hypothesize that the association between the reported signs and symptoms and the diagnosis of mutations or variants in the $A C A D S$ gene could be coincidental. As a first step to study this, we searched the database of the laboratory for metabolic diseases in our center for the most frequently reported symptoms of patients for whom metabolic studies were requested between November 2007 and November 2008. Developmental delay was by far the most frequently reported, followed by behavioral disorders, epilepsy, hypotonia, and hypoglycemia. The incidence of these symptoms is comparable with that in the SCADD patient group (Fig. 1), suggesting at least that there is no specific cluster of clinical signs and symptoms in SCADD and that the association might indeed be coincidental.

\section{Implications with respect to policy toward SCADD patients and families}

The probable lack of clinical significance of SCADD has important implications for the clinical management and counselling of SCADD patients and families. First, the diagnosis of SCADD should never preclude a full diagnostic workup for other potential causes of the symptoms. Any delay in doing so, might postpone the diagnosis of other potentially treatable causes. Second, patients and parents, as well as their physicians, should be clearly informed about the potential lack of clinical relevance of the detected biochemical and genetic abnormalities concerning SCADD once the diagnosis has been made. Furthermore, without a clear clinical phenotype of SCADD to date, SCADD is not an optimal candidate for inclusion in newborn screening programs. Meanwhile, further studies are needed to fully unravel the implications of SCADD in order to answer the question whether SCADD is involved in a multifactorial disease or represents a nondisease.

Open Access This article is distributed under the terms of the Creative Commons Attribution Noncommercial License which permits any noncommercial use, distribution, and reproduction in any medium, provided the original author(s) and source are credited.

\section{References}

Amendt BA, Greene C, Sweetman L, Cloherty J, Shih V, Moon A, Teel L, Rhead WJ (1987) Short-chain acyl-coenzyme a dehydrogenase deficiency. Clinical and biochemical studies in two patients. J Clin Invest 79:1303-1309

Baerlocher KE, Steinmann B, Aguzzi A, Krahenbuhl S, Roe CR, Vianey-Saban C (1997) Short-chain acyl-CoA dehydrogenase deficiency in a 16-year-old girl with severe muscle wasting and scoliosis. J Inherit Metab Dis 20:427-431

Bhala A, Willi SM, Rinaldo P, Bennett MJ, Schmidt-Sommerfeld E, Hale DE (1995) Clinical and biochemical characterization of short-chain acyl-coenzyme a dehydrogenase deficiency. J Pediatr 126:910-915

Birkebaek NH, Simonsen H, Gregersen N (2002) Hypoglycaemia and elevated urine ethylmalonic acid in a child homozygous for the short-chain acyl-CoA dehydrogenase $625 \mathrm{G}>\mathrm{A}$ gene variation. Acta Paediatr 91:480-482

Bok LA, Vreken P, Wijburg FA, Wanders RJ, Gregersen N, Corydon MJ, Waterham HR, Duran M (2003) Short-chain Acyl-CoA dehydrogenase deficiency: studies in a large family adding to the complexity of the disorder. Pediatrics 112:1152-1155

Coates PM, Hale DE, Finocchiaro G, Tanaka K, Winter SC (1988) Genetic deficiency of short-chain acyl-coenzyme a dehydrogenase in cultured fibroblasts from a patient with muscle carnitine deficiency and severe skeletal muscle weakness. J Clin Invest 81:171-175

Corydon MJ, Vockley J, Rinaldo P, Rhead WJ, Kjeldsen M, Winter V, Riggs C, Babovic-Vuksanovic D, Smeitink J, De Jong J, Levy H, Sewell AC, Roe C, Matern D, Dasouki M, Gregersen N (2001) Role of common gene variations in the molecular pathogenesis of shortchain acyl-CoA dehydrogenase deficiency. Pediatr Res 49:18-23

Dietzen DJ, Rinaldo P, Whitley RJ, Rhead WJ, Hannon WH, Garg UC, Lo SF, Bennett MJ (2009) National academy of clinical biochemistry laboratory medicine practice guidelines: follow-up testing for metabolic disease identified by expanded newborn screening using tandem mass spectrometry; executive summary. Clin Chem 55:1615-1626

Dutch Hospital Database, 2009. http://cognosserver.prismant.nl/ cognos 7/cgi-bin/ppdscgi.cgi?DC=Q\&E=/Prisma-LandelijkeLMR/Landelijke+LMR-informatie+-+Diagnosen. 
Gregersen N, Andresen BS, Bross P (2000) Prevalent mutations in fatty acid oxidation disorders: diagnostic considerations. Eur J Pediatr 159(Suppl 3):S213-S218

Gregersen N, Andresen BS, Pedersen CB, Olsen RK, Corydon TJ, Bross P (2008) Mitochondrial fatty acid oxidation defectsremaining challenges. J Inherit Metab Dis 31:643-657

Gregersen N, Winter VS, Corydon MJ, Corydon TJ, Rinaldo P, Ribes A, Martinez G, Bennett MJ, Vianey-Saban C, Bhala A, Hale DE, Lehnert W, Kmoch S, Roig M, Riudor E, Eiberg H, Andresen BS, Bross P, Bolund LA, Kolvraa S (1998) Identification of four new mutations in the short-chain acyl-CoA dehydrogenase (SCAD) gene in two patients: one of the variant alleles, $511 \mathrm{C} \rightarrow \mathrm{T}$, is present at an unexpectedly high frequency in the general population, as was the case for $625 \mathrm{G}->\mathrm{A}$, together conferring susceptibility to ethylmalonic aciduria. Hum Mol Genet 7:619-627

Jethva R, Ficicioglu C (2008) Clinical outcomes of infants with shortchain acyl-coenzyme a dehydrogenase deficiency (SCADD) detected by newborn screening. Mol Genet Metab 95:241-242

Jethva R, Bennett MJ, Vockley J (2008) Short-chain acyl-coenzyme a dehydrogenase deficiency. Mol Genet Metab 95:195-200

Jungbluth H (2007) Multi-minicore Disease. Orphanet J Rare Dis 2:31

Kmoch S, Zeman J, Hrebicek M, Ryba L, Kristensen MJ, Gregersen N (1995) Riboflavin-responsive epilepsy in a patient with SER209 variant form of short-chain acyl-CoA dehydrogenase. J Inherit Metab Dis 18:227-229

Koeberl DD, Young SP, Gregersen NS, Vockley J, Smith WE, Benjamin DK Jr, An Y, Weavil SD, Chaing SH, Bali D, McDonald MT, Kishnani PS, Chen YT, Millington DS (2003) Rare disorders of metabolism with elevated butyryl-and isobutyryl-carnitine detected by tandem mass spectrometry newborn screening. Pediatr Res 54:219-223

Kristensen MJ, Kmoch S, Bross P, Andresen BS, Gregersen N (1994) Amino acid polymorphism (Gly209Ser) in the ACADS gene. Hum Mol Genet 3:1711

Kurian MA, Hartley L, Zolkipli Z, Little MA, Costigan D, Naughten ER, Olpin S, Muntoni F, King MD (2004) Short-chain acyl-CoA dehydrogenase deficiency associated with early onset severe axonal neuropathy. Neuropediatrics 35:312-316

Maldegem BT, Waterham HR, Duran M, Vlies M, Woerden CS, Bobu LL, Wanders RJ, Wijburg FA (2005) The 625G $>$ A SCAD gene variant is common but not associated with increased C4-carnitine in newborn blood spots. J Inherit Metab Dis 28:557-562

Matern D, Hart P, Murtha AP, Vockley J, Gregersen N, Millington DS, Treem WR (2001) Acute fatty liver of pregnancy associated with short-chain acyl-coenzyme a dehydrogenase deficiency. J Pediatr 138:585-588

Nagan N, Kruckeberg KE, Tauscher AL, Bailey KS, Rinaldo P, Matern D (2003) The frequency of short-chain acyl-CoA dehydrogenase gene variants in the US population and correlation with the C(4)-acylcarnitine concentration in newborn blood spots. Mol Genet Metab 78:239-246

Naito E, Indo Y, Tanaka K (1990) Identification of two variant short chain acyl-coenzyme a dehydrogenase alleles, each containing a different point mutation in a patient with short chain acyl-coenzyme a dehydrogenase deficiency. J Clin Invest 85:1575-1582

National Newborn Screening Status Report (2008) Updated 07/07/09. National Newborn Screening and Genetics Resource Center (NNSGRC) Austin, Texas USA. http:/genes-r-us.uthscsa.edu/ nbsdisorders.pdf.

Pedersen CB, Kolvraa S, Kolvraa A, Stenbroen V, Kjeldsen M, Ensenauer R, Tein I, Matern D, Rinaldo P, Vianey-Saban C, Ribes A, Lehnert W, Christensen E, Corydon TJ, Andresen BS, Vang S, Bolund L, Vockley J, Bross P, Gregersen N (2008) The ACADS gene variation spectrum in 114 patients with short-chain acyl-CoA dehydrogenase (SCAD) deficiency is dominated by missense variations leading to protein misfolding at the cellular level. Hum Genet 124:43-56

Rhead WJ, Allain D, Van Calcar S, Hanson K, Wolff J, Larson CA, Zytkovicz TH, Levy H, Waisbren S, Shih VE, Marsden D (2002) Short-chain acyl-coenzyme a dehydrogenase (SCAD) and 3Methylcrotonyl-CoA carboxylase (MCC) deficiencies: tandem mass spectrometry newborn screening detects many clinically benign cases. J Inherit Metab Dis 25(Suppl1):4

Seidel J, Streck S, Bellstedt K, Vianey-Saban C, Pedersen CB, Vockley J, Korall H, Roskos M, Deufel T, Trefz KF, Sewell AC, Kauf E, Zintl F, Lehnert W, Gregersen N (2003) Recurrent vomiting and ethylmalonic aciduria associated with rare mutations of the short-chain acyl-CoA dehydrogenase gene. J Inherit Metab Dis 26:37-42

Tein I, Elpeleg O, Ben-Zeev B, Korman SH, Lossos A, Lev D, LermanSagie T, Leshinsky-Silver E, Vockley J, Berry GT, Lamhonwah AM, Matern D, Roe CR, Gregersen N (2008) Short-chain acyl-CoA dehydrogenase gene mutation $($ c. $319 \mathrm{C}>\mathrm{T})$ presents with clinical heterogeneity and is candidate founder mutation in individuals of Ashkenazi Jewish origin. Mol Genet Metab 93:179-189

van Maldegem BT, Duran M, Wanders RJ, Niezen-Koning KE, Hogeveen M, Ijlst L, Waterham HR, Wijburg FA (2006) Clinical, biochemical, and genetic heterogeneity in short-chain acylcoenzyme a dehydrogenase deficiency. JAMA 296:943-952

Waisbren SE, Levy HL, Noble M, Matern D, Gregersen N, Pasley K, Marsden D (2008) Short-chain acyl-CoA dehydrogenase (SCAD) deficiency: an examination of the medical and neurodevelopmental characteristics of 14 cases identified through newborn screening or clinical symptoms. Mol Genet Metab 95:39-45

Wilcken B (2008) The consequences of extended newborn screening programmes: do we know who needs treatment? J Inherit Metab Dis doi: $10.1007 / \mathrm{s} 10545-008-0843-8$ 